\title{
Aquaporin-4-antibody-seronegative neuromyelitis optica is less common than previously thought
}

New data indicate that many patients with neuromyelitis optica (NMO) who are apparently seronegative for aquaporin-4 autoantibodies (AQP4-Ab) either have another underlying condition or are found to be seropositive when assessed using next-generation assays.

$\mathrm{NMO}$ is an autoimmune inflammatory disease that affects the optic nerve and spinal cord, often leading to blindness and motor disability. To help distinguish NMO from other neuroinflammatory diseases, patients' serum is screened for AQP4-Ab. Traditionally, such screening has been carried out using tissue-based indirect immunofluorescence (IIF), which is highly specific but not very sensitive.

Sean Pittock's team at the Mayo Clinic reassessed the sera of 49 IIFseronegative patients with NMO using highly sensitive recombinant-antigenbased assays. $61 \%$ of these patients were shown to be seropositive when tested with the contemporary assays. The overall
4 Assay insensitivity overestimates the frequency of seronegativity and invalidates phenotypic comparisons... 77

proportion of seropositive patients in this centre increased from $67 \%$ to $88 \%$, indicating that genuinely $\mathrm{AQP} 4-\mathrm{Ab}$ seronegative NMO is uncommon.

"Assay insensitivity overestimates the frequency of seronegativity and invalidates phenotypic comparisons," write Pittock and colleagues. As they also note, another possible confounding factor is conversion from seropositive to seronegative during immunosuppressive therapy.

The results of a second study, led by Jacqueline Palace from Oxford University Hospitals, suggest that an alternative diagnosis-such as systemic infection or acute disseminated encephalomyelitiscan be made for many patients thought to have AQP4-Ab-seronegative NMO. Taken together, these two reports indicate that previous studies may have misdiagnosed a high number of patients as having seronegative NMO.

Attack severity and disability outcome did not differ between seropositive and seronegative patients in these two studies. According to Pittock's team, however, a population-based study is needed to yield enough truly seronegative patients to enable reliable comparisons with seropositive patients. Treatment with immunosuppressants was associated with a decreased relapse rate in both groups, highlighting the importance of immunosupressive therapy.

\section{Hemi Malkki}

Original article Jiao, Y. et al. Updated estimate of AQP4-IgG serostatus and disability outcome in neuromyelitis optica. Neurology doi:10.1212/WNL.0b013e3182a6cb5c

Further reading Kitley, J. et al. Longitudinally extensive transverse myelitis with and without aquaporin 4 antibodies. JAMA Neurol. doi:10.1001/jamaneurol.2013.3890 